\title{
Efecto de la situación sanitaria del galpón de gallinas sobre los estadios larvarios de endoparásitos en los hospederos intermediarios y en el comportamiento productivo
}

\author{
EFFECT OF THE SANITARY SITUATION OF THE POULTRY HOUSE ON THE LARVAL STAGES OF \\ ENDOPARASITES IN THE INTERMEDIATE HOSTS AND ON THE PRODUCTIVE PERFORMANCE
}

Gabriel Gorrín A. ${ }^{2}$, Manuel Colas C., ${ }^{1,3}$, Teresa Meireles R. ${ }^{1}$, Edmundo O. Pérez R. ${ }^{1}$

\section{Resumen}

El objetivo del estudio fue evaluar el efecto de la situación sanitaria de los galpones de gallinas en la prevalencia de los estadios larvarios de endoparásitos en los hospederos intermediarios y en el comportamiento productivo en gallinas ponedoras White Leghorn de 55 semanas durante ocho semanas. Se diseñaron dos tratamientos (T1 y T2) con 486 gallinas cada uno (tres réplicas de 162 aves), según la calidad higiénica de dos galpones (niveles de polvo en el suelo, encharcamiento en fosos de deyecciones, plumas y huevos rotos en suelo y pasillos, presencia de aves silvestres, población de insectos). Cada 15 días (cuatro muestreos) se recolectó $1 \mathrm{~kg}$ de excretas por réplica de las torres de deyecciones para cuantificar y clasificar los hospederos intermediarios. Se identificaron todos los insectos y artrópodos y se cuantificó la presencia de larvas de nematodos y quistes larvarios. Se recolectaron los intestinos de 15 aves $(\mathrm{T} 1=7 ; \mathrm{T} 2=8)$ para la identificación de endoparásitos. Se determinó la extensión de la infestación y se estimaron las variables productivas (producción de huevos total y por ave, porcentaje de postura, conversión, viabilidad y calidad externa del huevo). Los resultados indicaron que la situación sanitaria del galpón con deficientes condiciones higiénicas (T2) influyó positivamente en las poblaciones de hospederos intermediarios de cestodos y en una mayor producción de huevos con cáscara sucia, manchada y rota. Alphitobius diaperinus fue el coleóptero de mayor presentación y con mayor intensidad de infestación por Subulura suctoria.

Palabras clave: estadios larvarios; endoparásitos; Subulura suctoria; hospederos intermediarios; Alphitobius diaperinus; Forficula auricularia

\footnotetext{
${ }^{1}$ Facultad de Medicina Veterinaria, Universidad Agraria de la Habana, Cuba

${ }^{2}$ Entidad de Ciencia, Tecnología e Innvación - ECTI, Sierra Maestra, Cuba

${ }^{3}$ E-mail: manuelcc@unah.edu.cu
}

Recibido: 21 de septiembre de 2017

Aceptado para publicación: 12 de abril de 2018 
The objective of the study was to evaluate the effect of the health status of poultry houses on the prevalence of larval stages of endoparasites in intermediate hosts and on the productive behavior of 55-week-old White Leghorn laying hens for eight weeks. Two treatments were designed (T1 and T2) with 486 chickens each (three replicas of 162 birds), according to the hygienic quality of two chicken houses (dust levels in the floor, puddles in excreta towers, feathers and broken eggs in the floor and corridors, presence of wild birds, population of insects). Every 15 days (four samplings) $1 \mathrm{~kg}$ of excreta per replica was collected from the excreta towers to quantify and classify the intermediate hosts. All insects and arthropods were identified, and the presence of nematode larvae and larval cysts was quantified. The intestines of 15 birds $(\mathrm{T} 1=7 ; \mathrm{T} 2=8)$ were collected for the identification of endoparasites. The extension of the infestation was determined, and the productive variables were estimated (production of total eggs and per bird, percentage of egg laying, conversion, viability, and external quality of the egg). The results indicated that the sanitary situation of the chicken house with poor hygienic conditions (T2) positively influenced the populations of intermediary hosts of cestodes and in a greater production of eggs with dirty, stained, and broken shells. Alphitobius diaperinus was the coleopter with the highest presentation and with the highest intensity of infestation by Subulura suctoria.

Keywords: larval states; endoparasite; Subulura suctoria; intermediate hosts; Alphitobius diaperinus; Forficula auricularia

\section{INTRODUCCIÓN}

En las granjas avícolas y otras de producción pecuaria, el manejo de las excretas se puede convertir en un problema sanitario si no se encuentran los mecanismos adecuados para procesarlas (Lemus, 2016). La gran cantidad de nutrientes que se encuentran en la gallinaza propicia un microhábitat ideal para el establecimiento de fauna entomológica, como insectos y ácaros; tanto aquellos artrópodos degradadores de materia orgánica como Caloglyphus sp y los depredadores de otros organismos como Macrocheles sp, además de insectos que funcionan como reservorios de parásitos de las gallinas, como la mosca Musca domestica y el tenebriónido Alphitobius diaperinus (Vergara et al., 2013). La importancia económica de los coleópteros radica en las grandes pérdidas que ocasionan en las aves al causar estrés, inapetencia, retraso en el crecimiento y baja producción (Rodríguez et al., 2013), siendo
A. diaperinus uno de los principales en explotaciones avícolas (Domínguez, 2012).

La presencia de hospederos intermediarios para los parásitos y la disminución de la efectividad de algunos antihelmínticos contribuyen a que existan infecciones parasitarias graves en las aves (Menéndez, 2007; Madrigal et al., 2008).

En las nematodosis, las especies más relevantes son Subulura suctoria y $S$. brumpti, que se caracterizan por ser pequeños, finos, blanquecinos y parasitan el ciego de la gallina doméstica, la gallina de Guinea y los pavos. Desarrollan un ciclo biológico indirecto, utilizando como hospederos intermediarios a los coleópteros $A$. diaperinus y Dermestes ater. Los huevos de estos nematodos se eliminan con las heces y son ingeridas por el hospedero intermediario donde la larva se libera, atraviesa la pared intestinal y se desarrolla en la cavidad corporal para ser larva infectante (tercer estadio) en aproxi- 
madamente dos semanas, donde permanecen hasta que las aves ingieren el coleóptero (Sánchez y Lamazares, 2010; Roque, 2015).

Las prácticas de crianza modernas impiden el contacto de los cestodos con el hospedero intermediario por lo que se rompe el ciclo y, además, se dispone de cesticidas efectivos que explican la menor afectación. Parásitos de los géneros Raillietina y Choanotaenia incluyen los cestodos grandes, pudiendo llegar a medir hasta $25 \mathrm{~cm}$, pero se requiere de la microscopía para su identificación diagnóstica basándose en la diferencia de los proglótidos (Roque, 2015).

El cestodo Choanotaenia infundibulum se encuentra en todas las provincias de Cuba y se ha diagnosticado solo y asociado a Raillietina cesticillus y $R$. tetragona; sin embargo, $R$. cesticillus y $C$. infundibulum son las especies que más afectan a la gallina doméstica y que requieren del coleóptero $D$. ater como su hospedero intermediario (Hernández et al., 2002). Según Sánchez y Lamazares (2010), las especies $R$. cesticillus, $R$. tetragona y $R$. echinobotrida son las de mayor presentación en la gallina doméstica dentro del género Raillietina. Los proglótidos maduros se desprenden del cuerpo del parásito y salen con las heces, donde son ingeridos por el hospedero intermediario y evolucionan hasta cisticercoide. Este se libera cuando el hospedero intermediario es ingerido por las aves y se fija en la mucosa del intestino desarrollándose hasta adulto. Además, los referidos autores destacan que no se presentan infecciones en la crianza en jaulas, con excepción de Choanotaenia.

Menéndez (2007) refiere que es imposible evitar la ingestión de los hospederos intermediarios por las aves, de allí que se debe disminuir el número de portadores de cisticercoides, retirando el estiércol del local y espolvoreando el excremento con insecticidas. El control sistemático contra los hospederos intermediarios es una cuestión fundamental en la lucha contra los nematodos aviares (Madrigal et al., 2008). El presente trabajo tuvo como objetivo evaluar el efecto de la situación sanitaria del galpón de gallinas sobre los estadios larvarios de endoparásitos en los hospederos intermediarios y en el comportamiento productivo.

\section{Materiales y Métodos}

El estudio se realizó en una granja de ponedoras perteneciente a la empresa avícola de Artemisa, ubicada en la región occidental de Cuba. Se utilizaron gallinas L33 White Leghorn de 55 semanas y con sistema de crianza en batería. El experimento tuvo una duración de ocho semanas durante los meses de abril y mayo, correspondientes al periodo poco lluvioso e inicio del periodo lluvioso, respectivamente.

Las aves se encontraron bajo las mismas condiciones de tenencia, manejo y alimentación. Los tratamientos se formaron según la calidad higiénica de los galpones de crianza indicada en el Cuadro 1. Se consideraron los términos 'escaso' y 'abundante' para definir que las variables en estudio se encontraban presente en una cantidad inferior o superior a la que es habitual encontrar en los galpones, respectivamente, mientras que el término 'moderado' se incluyó como una clasificación intermedia entre los adjetivos escaso y abundante.

Las aves se dividieron en dos grupos experimentales formados por tres réplicas cada una de 162 aves, dando un total de 486 gallinas por tratamiento. Tres aves estuvieron alojadas por celda.

\section{Esquema de Muestreo Parasitológico}

- Muestreo de excretas para cuantificar e identificar los hospederos intermediarios. Se recolectó $1 \mathrm{~kg}$ de excretas por réplica divididas en 5 submuestras de 200 $\mathrm{g}$ de las torres de deyecciones cada 15 días en 4 oportunidades, para cuantificar y clasificar los hospederos intermediarios 
en coleópteros y tijeretas. Para la identificación de los dermápteros y coleópteros se utilizaron las claves descritas por Michel (2006) y Pegorini (2016). Para la cuantificación, se separaron en grupos y se colocaron en frascos de $100 \mathrm{ml}$. Se les proveyó con $15 \mathrm{~g}$ de alimento (pienso, pan y pequeñas porciones de excretas) para preservar la viabilidad durante $24 \mathrm{~h}$ hasta su traslado al Laboratorio de Patología de la Universidad Agraria de la Habana (UNAH).

- Identificación de las larvas de nematodos y quistes larvarios en los hospederos intermediarios. Todos los hospederos intermediarios, conservados en frascos de $100 \mathrm{ml}$ por réplica de cada tratamiento en los 4 muestreos, fueron inmovilizados y mediante el método de compresión, auxiliados por pinzas y agujas entomológicas, se les extrajo el contenido de la cavidad corporal para identificar quistes larvarios de cestodos y larvas de nematodos. Para la identificación de larvas de nematodos en los coleópteros se utilizó la clave descrita por Barus (1970).

- Intensidad de infestación de larvas y quistes larvarios. Se cuantificaron las larvas y quistes larvarios encontrados, así como el número de hospederos intermediarios positivos y negativos a los mismos.

- Recolección de intestinos. Siete gallinas de T1 y 8 gallinas de T2 fueron sacrificadas. La necropsia se realizó siguiendo la metodología descrita por Colas et al. (2010) y se extrajeron los intestinos para la recolección de los endoparásitos. Las muestras se conservaron en congelación para facilitar el desprendimiento del escólex de los parásitos de la mucosa intestinal.

- Interpretación de los resultados. La extensión de infestación por tratamiento se expresó en forma porcentual ([coleópteros positivos/total de coleópteros] x 100) y la intensidad de infestación estuvo referida al total de larvas por coleóptero.

\section{Variables Productivas}

Se consideraron la producción de huevos totales, huevos por ave, porcentaje de postura, conversión ( $\mathrm{kg} / 10$ huevos), calidad externa del huevo (sanos, sucios, cascados, manchados y rotos) y mortalidad por réplica y tratamiento. Además, se realizó una anamnesis epidemiológica e inspección clínica individual y colectiva de las gallinas y se realizaron las necropsias en casos de muerte.

\section{Análisis Estadístico}

Se realizó un análisis de varianza de clasificación simple teniendo en cuenta los indicadores de mortalidad, producción de huevos totales, huevo/ave, porcentaje de postura y conversión utilizando el paquete estadístico Statgraphics Plus 5.1. Para evaluar la calidad externa de los huevos y mortalidad por causa entre tratamientos se utilizó el COMPROP 1.

\section{Resultados y Discusión}

En la Figura 1 se aprecia que las poblaciones de hospederos intermediarios no presentaron variaciones en $\mathrm{T} 2$ entre el primer y segundo muestreo, mientras que en las poblaciones de hospederos intermediarios en $\mathrm{T} 1$ fueron inferiores a las encontradas en el T2, especialmente en el tercer muestreo. Se encontraron diferencias significativas en la proporción de coleópteros entre los dos galpones de crianza, más no así para las tijeretas (Cuadro 2).

Entre los factores que pudieron haber influido en una menor cantidad de coleópteros en T1, Quintero et al. (2015) plantean que se debe considerar el papel de las tijeretas como depredadores de estos insectos, aspecto esencial a la hora de relacionarlas con el número de coleópteros, ya que estas se alimentan de sus larvas y pudieran funcionar como control biológico de los mismos. 
Cuadro 1. Características de higiene de los galpones de crianza del estudio

\begin{tabular}{lll}
\hline & T1 (nave 1) & T2 (nave 2) \\
\hline $\begin{array}{l}\text { Niveles de polvo en el suelo } \\
\begin{array}{l}\text { Encharcamiento en fosos de } \\
\text { deyecciones }\end{array}\end{array}$ & Escasos & Abundantes \\
Torres de excretas & $\begin{array}{l}\text { Abundantes con altura } \\
\text { elevada }\end{array}$ & $\begin{array}{l}\text { Escasas y con baja } \\
\text { altura }\end{array}$ \\
$\begin{array}{l}\text { Plumas y huevos rotos en suelo y } \\
\text { pasillos }\end{array}$ & Ausentes & Abundantes \\
$\begin{array}{l}\text { Presencia de aves silvestres } \\
\text { Población de insectos }\end{array}$ & Pocas & Muchas \\
\hline
\end{tabular}

Cuadro 2. Análisis de proporciones entre los coleópteros y las tijeretas entre galpones de crianza (tratamientos ${ }^{1}$ )

\begin{tabular}{lccc}
\hline $\begin{array}{l}\text { Hospedero } \\
\text { intermediario }\end{array}$ & \multicolumn{2}{c}{ Tratamiento Proporción } & $\begin{array}{c} \pm \\
\text { EE }\end{array}$ \\
\hline \multirow{2}{*}{ Coleópteros } & $\mathrm{T} 1$ & $0.38^{\mathrm{a}}$ & \\
& $\mathrm{T} 2$ & $0.62^{\mathrm{b}}$ & 0.02 \\
\multirow{2}{*}{ Tijeretas } & $\mathrm{T} 1$ & $0.52^{\mathrm{a}}$ & \\
& $\mathrm{T} 2$ & $0.48^{\mathrm{a}}$ & 0.03 \\
\hline
\end{tabular}

${ }^{a, b}$ Letras diferentes dentro de hospederos indica diferencia significativa $(p<0.001)$

${ }^{1}$ Ver Cuadro 1

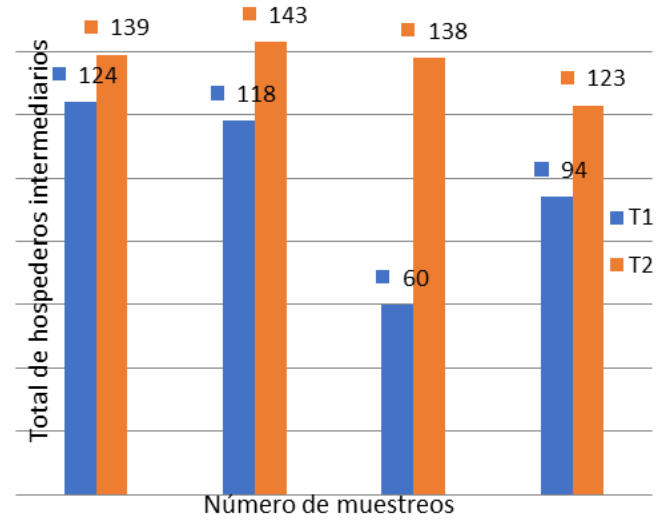

Figura 1. Comportamiento de las poblaciones de hospederos intermediarios por tratamiento en los cuatro muestreos

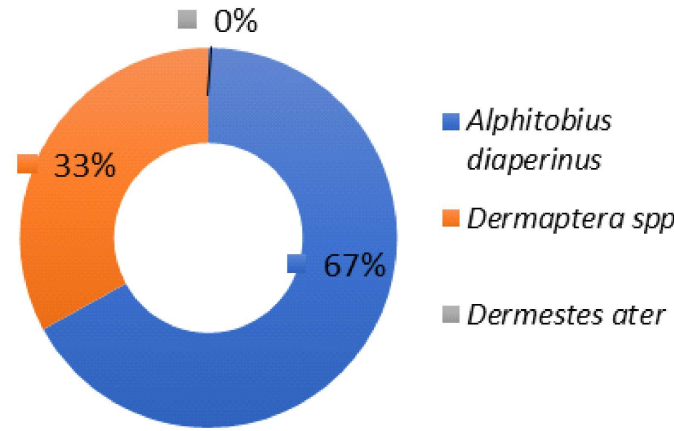

Figura 2. Especies de artrópodos e insectos hospederos intermediarios de parásitos de aves encontrados durante el estudio

Vergara et al. (2013) comentan que en la materia orgánica se pueden localizar artrópodos de hábitos variados, así como insectos que funcionan como reservorios de agentes etiológicos de enfermedades y parásitos de las gallinas, como es el caso de la mosca Musca domestica y del tenebriónido $A$. diaperinus. En la Figura 2 se observa que la especie predominante durante el experimento fue $A$. diaperinus, representando el $67 \%$ de las especies encontradas. Estos resultados fueron similares al trabajo de Retamares et al. (2011) quienes encuentran $A$. diaperinus como el insecto más frecuente $(74.6 \%)$ en excretas. Por otra parte, Quintero et al.(2015) 
Cuadro 3. Indicadores de extensión (E.I.) e intensidad (I.I.) de infestación en coleópteros positivos a estadios larvarios L3 de Subulura suctoria en granjas de ponedoras

\begin{tabular}{ccc}
\hline Tratamientos $^{1}$ & E.I. (\%) & I.I. \\
\hline T1 & 6.72 & 2.18 \\
T2 & 5.31 & 3.00 \\
\hline
\end{tabular}

${ }^{1}$ Ver Cuadro 1

Cuadro 4. Análisis de los indicadores productivos entre tratamientos ${ }^{1}$ en aves ponedoras

\begin{tabular}{lccc}
\hline Indicadores & Tratamiento & Media & $\begin{array}{c} \pm \\
\text { EE }\end{array}$ \\
\hline Prod. de & 1 & $310.14^{\mathrm{A}}$ & 1.93 \\
huevos (n) & 2 & $296.12^{\mathrm{B}}$ & \\
Huevo/ave & 1 & $0.63^{\mathrm{a}}$ & \\
$(\mathrm{n})$ & 2 & $0.60^{\mathrm{b}}$ & 0.00 \\
Postura & 1 & $63.81^{\mathrm{A}}$ & \\
$(\%)$ & 2 & $60.93^{\mathrm{B}}$ & 0.39 \\
$\begin{array}{l}\text { Conversión } \\
\text { (kg/10 }\end{array}$ & 1 & $1.75^{\mathrm{A}}$ & \\
huevos) & 2 & $1.67^{\mathrm{B}}$ & 0.01 \\
\hline
\end{tabular}

Letras diferentes $\left({ }^{A, B} y^{a, b}\right)$ dentro de indicadores indican diferencia significativa $(p<0.001, p<0.01$, respectivamente)

${ }^{1}$ Ver Cuadro 1

reportan $0.1 \%$ de $A$. diaperinus y Forficula auricularia en gallinaza de granjas de ponedoras en México. Cecco et al. (2005) plantean que el ambiente húmedo y cálido favorece el desarrollo del ciclo biológico de $A$. diaperinus, ya que se ve atraído por el NH3.

El indicador de extensión de infestación fue superior en $\mathrm{T} 1$ respecto a $\mathrm{T} 2$, pero el indicador de intensidad de infestación fue menor (Cuadro 3), posiblemente debido a que en $\mathrm{T} 1$, al existir un menor número de coleópteros como consecuencia de una mejor higiene en los galpones de crianza y una mayor presentación de tijeretas, son menores las probabilidades de infestación de $S$. suctoria. La proporción de este cestode fue de $38 \%$ en $\mathrm{T} 1$ y de $62 \%$ en T2 ( $<<0.001)$.

En la identificación de larvas de nematodos y quistes larvarios de cestodos en los hospederos intermediarios en los cuatro muestreos solo se encontraron larvas L3 de $S$. suctoria en varios ejemplares de $A$. diaperinus, en tanto que todas las tijeretas resultaron negativas a larvas y quistes larvarios. Los resultados coinciden con otros estudios (Tamayo et al., 1991; Sánchez y Lamazares, 2010; Roque, 2015) que plantean que $S$. suctoria presenta ciclo biológico indirecto utilizando como hospederos intermediarios los coleópteros A. diaperinus y $D$. ater.

El análisis de los parámetros productivos se muestra en el Cuadro 4. Se observan mejores resultados en $\mathrm{T} 1$ debido a una mejor calidad sanitaria en la nave de crianza. La situación sanitaria deficiente en T2 permite un aumento de las poblaciones de coleópteros que producen estrés y malestar en las aves (Godwin y Waltman, 1996; Rodríguez et al., 2013).

No se encontraron parásitos en las necropsias. Respecto a la mortalidad, solo se produjo una muerte por prolapso en la semana 6 en T1, mientras que en T2 ocurrieron dos muertes por enterobacteriosis asociadas a otras enfermedades.

En el análisis de la calidad externa de los huevos se encontró una mayor cantidad de huevos sucios, manchados y rotos en T2 en comparación con $\mathrm{T} 1$ ( $\mathrm{p}<0.05$; Cuadro 5). La situación sanitaria tiene un efecto en las poblaciones de vectores y hospederos intermediarios que transmiten enterobacterias (Corredor et al., 2006). En el transcurso del estudio se observaron muchas aves con diarrea en T2 trayendo como consecuencia la producción de huevos sucios y manchados. 
Cuadro 5. Comparación de la calidad externa de los huevos por tratamiento ${ }^{1}$ en aves de postura

\begin{tabular}{lccc}
\hline $\begin{array}{l}\text { Calidad } \\
\text { externa }\end{array}$ & \multicolumn{2}{c}{ Tratamiento Proporción } & $\begin{array}{c} \pm \\
\text { EE }\end{array}$ \\
\hline Sanos & 1 & $0.51^{\mathrm{a}}$ & 0 \\
& 2 & $0.49^{\mathrm{b}}$ & \\
Sucios & 1 & $0.41^{\mathrm{a}}$ & 0.03 \\
& 2 & $0.59^{\mathrm{b}}$ & \\
Cascados & 1 & $0.46^{\mathrm{a}}$ & 0.03 \\
& 2 & $0.54^{\mathrm{a}}$ & \\
Manchados & 1 & $0.36^{\mathrm{a}}$ & 0.04 \\
& 2 & $0.64^{\mathrm{b}}$ & \\
Rotos & 1 & $0.29^{\mathrm{a}}$ & 0.05 \\
& 2 & $0.71^{\mathrm{b}}$ & \\
\hline
\end{tabular}

$a, b$ Letras diferentes dentro de calidades externas indican diferencias significativas $(p<0.001)$

${ }^{1}$ Ver Cuadro 1

\section{Conclusiones}

- La situación sanitaria del galpón con deficientes condiciones higiénicas (T2) influyó positivamente en las poblaciones de hospederos intermediarios de helmintos y en una mayor producción de huevos con cáscara sucia, manchada y rota.

- Alphitobius diaperinus fue el coleóptero de mayor presentación y con mayor intensidad de infestación de Subulura suctoria.

\section{Literatura Citada}

1. Barus V. 1970. Studies of the nematode Subulurasuctoria II. Development in the intermediate Host. Folia Parasitológica 7: 50-57.

2. Cecco L, González H, Deluchi P, Barrios H, De Franceschi M. 2005. Determinación de los estados de desarrollo de Alphitobius diaperinus en granjas avícolas. Rev Argentina Prod Anim 25: 93-99.

3. Colas M, García AJ, Merino A, Sánchez A, Corea A, Bacallao E, Mojena K, et al. 2010. Estudio de la anamnesis epizoótica y de la necropsia de aves domésticas en la base asistencial veterinaria. REDVET 11(11B). [Internet]. Disponible en: www.veterinaria.org/revistas/redvet/n111110B/ 111008B.pdf

4. Corredor CA, Hortua LC, Pinzon GE. 2006. Enfermedades parasitarias de las aves. Colombia: Universidad Pedagógica y Tecnológica de Colombia. [Internet]. Disponible en: http://patologiaaviaruptc.blogspot.com/2006/11/enfermedades-parasitarias-de-las-aves.html

5. Domínguez I. 2012. Alphitobius diaperinus ¿Un problema bajo control o bajo los comederos? Últimos avances en su control. Selecciones Avícolas 54(5): 23-27.

6. Godwin MA, Waltman WD. 1996. Transmission of Eimeria, viruses, and bacteria to chicks: darkling beetles (Alphitobius diaperinus) as vector of pathogens. J Appl Poultry Res 5: 51-55.

7. Hernández $M$, Larramendy $R$, $S z c z y p e l$ B. 2002. Céstodos más frecuentes de la gallina doméstica en Cuba. Rev Cubana Cienc Avícola 26: 145-149.

8. Lemus LA. 2016. Manejo de la gallinaza: compostar para lograr una granja libre de malos olores y moscas. El Sitio Avícola [Internet]. Disponible en: http:// www.elsitioavicola.com/articles/2913/ manejo-de-lagallinaza-compostar-paralograr una-granja-libre-de-malos-oloresy-moscas/

9. Madrigal W, Hernández A, Madrigal M, Quintana A. 2008. Nematodosis más frecuentes en las aves domésticas. Rev Cubana Cienc Avícola 32: 67-71.

10. Menéndez T. 2007. Prevalencia de nemátodos y céstodos en aves de corral (traspatio) en la ciudad de Acayucán, Ver. Tesis de Médico Veterinario. Veracruz, México: Univ. Veracruzana. 101 p. 
11. Michel J. 2006. Dermaptera de Nicaragua. Rev Nica Ent 66 (Supl 2): 1-127.

12. Pegorini C. 2016. Associasao do óleo esencial de Eugenia uniflora $E$ Bacillusthuringiensis sobre Alphitobius diaperinus (PANZER). Tesis de Maestría. Brazil: Universidade Tecnológica Federal do Paraná. $64 \mathrm{p}$.

13. Quintero MT, Mendoza A, Sosa FJ, Jasso A, Castilla G. 2015. Importancia de los escarabajos en explotaciones de aves. Sitio Avícola. [Internet]. Disponible en: http://www.elsitioavicola.com/ articles/2730/importancia-de-los-escarabajos-en-explotaciones-de-aves/

14. Retamales J, Vivallo F, Robeson J. 2011. Insects associated with chicken manure in a breeder poultry farm of Central Chile. Arch Med Vet 43: 79-83. doi: 10.4067/S0301-732X2011000100011

15. Rodríguez D, Suárez M, Merino A, Larramendy $R$, Temprana M, Díaz $O$, Morales Y, Rebollar R. 2013. Evaluación en condiciones de laboratorio de la eficacia de tres insecticidas de nuevo uso en la avicultura para el control de Alphitobius diaperinus. Rev Salud Anim 35: 197-200.

16. Roque E. 2015. Parasitología y enfermedades parasitarias en los animales domésticos. La Habana, Cuba: Félix Varela. 323 p.

17. Sánchez A, Lamazares MC. 2010. Principales enfermedades que afectan las aves. En: SánchezA, López A, García MC, Lamazares MC, et al (eds). Salud y producción de las aves. La Habana, Cuba: Félix Varela. p 414-514.

18. Tamayo Y, Fernández M, Cordovez CO, Vivanco J, Nuñez V, Rodríguez $\boldsymbol{R N}$. 1991. Efectividad y evaluación del mangle rojo (Rizophora mangle) contra el coleóptero Alphitobius diaperinus. Rev Cubana Cienc Avícola 18: 193-194.

19. Vergara S, Obregón JA, Núñez P, Soberanes N. 2013. Artrópodos asociados a gallinaza y plumas en aves de postura y engorde. Avicultores y su Entorno 94: 1313-1317. 